Article

\title{
Canopy Reflectance Modeling of Aquatic Vegetation for Algorithm Development: Global Sensitivity Analysis
}

\author{
Guanhua Zhou 1,2,3,*(D), Zhongqi Ma ${ }^{1}$, Shubha Sathyendranath ${ }^{2,4}$, Trevor Platt ${ }^{2,4}$, \\ Cheng Jiang ${ }^{5,6}$ and Kang Sun ${ }^{3}$ \\ 1 School of Instrumentation Science and Opto-electronics Engineering, Beihang Univerisity, \\ Beijing 100191, China; mzq11171079@163.com \\ 2 Plymouth Marine Laboratory, Prospect Place, Plymouth PL1 3DH, UK; ssat@pml.ac.uk (S.S.); \\ tplatt@pml.ac.uk (T.P.) \\ 3 CETC Key Laboratory of Aerospace Information Applications, Shijiazhuang 050081, China; \\ skgucas@163.com \\ 4 National Centre for Earth Observation, Plymouth Marine Laboratory, Prospect Place, \\ Plymouth PL1 3DH, UK \\ 5 Beijing Institute of Space Mechanics \& Electricity, Beijing 100094, China; cheng3515523@163.com \\ 6 Key Laboratory for Advanced Optical Remote Sensing Technology of Beijing, Beijing 100094, China \\ * Correspondence: zhouguanhua@buaa.edu.cn; Tel.: +86-010-82315884
}

Received: 28 April 2018; Accepted: 24 May 2018; Published: 27 May 2018

\begin{abstract}
Optical remote sensing of aquatic vegetation in shallow water is an essential aid to ecosystem protection, but it is difficult because the spectral characteristics of the vegetation are sensitive to external features such as water background effects, atmospheric effects, and the structural properties of the canopy. A global sensitivity analysis of an aquatic vegetation radiative transfer model provides invaluable background for algorithm development for use in optical remote sensing. Here, we use the extended Fourier Amplitude Sensitivity Test (EFAST) method for the modelling. Four different cases were identified by subdividing the ranges of water depth and leaf area index (LAI) involved. The results indicate that the reflectance of emergent vegetation is affected mainly by the concentrations of chlorophyll $\mathrm{a}+\mathrm{b}$ in leaves $(\mathrm{Cab})$, leaf inclination distribution function parameter (LIDFa) and LAI. The parameter LAI is influential in sparse vegetation cases whereas Cab and LIDFa are influential in dense vegetation cases. Canopy reflectance for submerged vegetation is dominated by water parameters. Relatively, LAI and Cab are highly sensitive vegetation parameters. The analysis is extended to vegetation index as well, which takes the Sentinel-2A as the reference sensor. It shows that NDAVI (Normalized Difference Aquatic Vegetation Index) is suitable for retrieving LAI in all cases except deep-sparse for emergent vegetation, whereas NDVI (Normalized Difference Vegetation Index) would be better in the deep-sparse case. NDVI, NDAVI and WAVI (Water Adjusted Vegetation Index), respectively, are suitable for retrieving Cab, Car and LIDFa in dense cases. For submerged vegetation, the sensitivity of LAI to NDAVI is relatively high only in the shallow-sparse case. The adjustment factor $L$ in SAVI and WAVI fails to suppress the sensitivity to water constituent parameters. The sensitivity of LAI and Cab to NDVI in deep cases is relatively higher than that to the other indices, which may provide clues for the construction of inversion algorithms in macrophyte remote sensing in the aquatic environment using spectral signatures in the visible and near infrared regions.
\end{abstract}

Keywords: aquatic vegetation; radiative transfer model; global sensitivity analysis; vegetation index; Sentinel-2A; wetland remote sensing 


\section{Introduction}

Aquatic vegetation is a key element in the sustainability of wetland ecosystems [1,2]. According to growth habitat, aquatic vegetation can be divided into three groups: emergent vegetation, whose roots grow in the sediment underwater but whose leaves and stems emerge out of water; floating vegetation, which has leaves floating just above the water surface; and submerged vegetation, which lives entirely below the water surface [3]. These plants serve to absorb nitrogen and phosphorus, reduce turbidity and inhibit eutrophication, and can be used as indicators of the trophic status of a water body. It is important to monitor the distribution of aquatic vegetation as an aid to protecting wetland ecosystems. Conventional field surveys of macrophyte communities are difficult to implement, requiring considerable time and expense to carry out over large spatial scales [4]. Even worse, traditional field-based surveys are commonly hindered by limited accessibility [5]. The technology of remote sensing has the advantage of a broad coverage and frequent data acquisitions, which makes it a valuable tool for the assessment of macrophyte distribution, vegetation status and related biophysical parameters.

At present, the technologies of remote sensing are widely used for mapping and classification of aquatic vegetation [6-8]. Another major application of wetland remote sensing is parameter inversion, which includes mainly biomass [9-12] and leaf area index (LAI) [13,14]. The inversion is usually based upon statistical methods that contain no fundamental mechanistic information about the optical properties of the vegetation itself. Moreover, the data required by the statistical methods are specific to the particular species in a particular region, which limits their accuracy when applied to other species or regions. It is mainly because of the complex growth environment, which has a significant impact on the spectral properties of the vegetation, that many scholars prefer statistical methods to mechanistically-based methods. As for emergent vegetation and floating vegetation, their spectra are similar to those of terrestrial vegetation with a green reflectance peak and "red edge", but the canopy reflectance will decrease with vegetation density as there will be more water exposed in the background causing attenuation, especially in the near-infrared (NIR) region [15]. Submerged vegetation is characteristic of deeper water. The strong absorption of water forces the canopy reflectance to be attenuated by a factor of order 100 [16]. In addition to water itself, optically active materials in the water (i.e., phytoplankton, pigments and yellow substances) can also affect the observed signal [17]. Thus, to improve the accuracy of inversion, it is imperative to understand the relative importance of interactions among the factors that influence reflectance in the macrophyte-water coupled system.

To achieve this goal, a radiative transfer model for aquatic vegetation is required. Exploration of the radiative transfer process and analyses of the reflectance spectra of aquatic vegetation have increased in recent years. Currently, there are several models available that are able to simulate the spectral response of aquatic vegetation [18-25]. We have proposed the Aquatic Vegetation Radiative Transfer model (AVRT) based on the SAIL model framework. The model stratifies the whole system and calculates the scattering matrix for each layer separately [26]. The bidirectional reflectance of the whole system is calculated after iterative accumulation. The AVRT model can calculate the spectra of emergent vegetation, submerged vegetation and floating vegetation in the band range of 400-1000 nm. This model is validated with in-situ spectral data and with a more accurate Monte Carlo model. By a holistic analysis of the sensitivity of reflectance, based on model simulation, we have been able to obtain a better understanding of the interactions between environmental factors and aquatic vegetation. Global sensitivity analysis is an excellent method to quantify the relative importance of each input variable to model output, and therefore to identify the key determinants of output such as reflectance. It can also help set reasonable default values under a relatively wide range of conditions for less influential variables [27]. Then we can try to retrieve the key parameters of wetlands from field spectra or images, and develop new approaches to extract more optical information about various wetland species. This work can facilitate the dynamic monitoring of wetlands and, finally, can benefit the protection of wetland ecosystems. 
The law of parsimony says that only a few input variables can account for most of the effects on the output response, so identifying these important parameters is essential for complex models with many input variables. Sensitivity analysis can be an effective tool to discriminate the effects of the models' various parameters [28]. Sensitivity analysis refers to calculation of how the uncertainties in a model's output should be assigned, qualitatively or quantitatively, to uncertainties in the various inputs. Generally, it can be classified into local sensitivity analysis and global sensitivity analysis. Local sensitivity analysis methods are often referred to as "one-factor-at-a-time" (OAT), because they change the value of one variable at a time while holding all others at their central values, then measuring variation in the outputs. Local sensitivity analysis can analyze the impact of only a single input parameter on the outputs and does not encompass the entire input variable space [29]. Thus, local sensitivity analysis methods are inadequate for analyzing complex models, with many variables, that may be highly dimensional as well as non-linear. However, global sensitivity analysis explores the full input variable space and can elucidate not only the main effect of each parameter but also the secondary or higher order interactions among parameters [28]. The commonly used global sensitivity analysis methods include correlation-based methods [30], variance decomposition methods [31] and density-based methods [32]. Sensitivity analysis has been widely used in various regimes [28]. Recently, some scholars have used global sensitivity analysis to discuss the properties of the models implemented in remote sensing [33,34]. Several papers in which sensitivity analysis is used for research on terrestrial vegetation also deserve attention. Xiao et al. analyzed the well-known canopy model Prosail by the extended Fourier Amplitude Sensitivity Test (EFAST) method. The analysis showed that LAI had the most significant effect on canopy reflectance for sparse canopy with LAI in the range of 0-3. For dense canopies (LAI =3-6), the concentrations of Chlorophyll a and $b$ in leaves were the most influential parameters in the visible region [35]. Mousivand et al. obtained the sensitivity not only of the canopy reflectance but also the Top-Of-Atmosphere (TOA) radiance of a soil-vegetation system, and the analyzed factors, including the biophysical and biochemical parameters of a coupled Soil-Leaf-Canopy model and Modtran. They found that crown coverage, LAI, leaf inclination distribution function (LIDF) and soil moisture were the most influential parameters in the range 400-2500 $\mathrm{nm}$ [36]. Verelst et al. used machine-learning methods to simplify several radiative transfer models (i.e., Prospect-4, Prosail, and Modtran-5). The simplified versions had the same sensitivity patterns as the original ones. Among them, the results of Modtran-5 showed that aerosol optical parameters such as AOT and Angstrom Coefficient had very significant effects in the range 400-2500 $\mathrm{nm}$ [37]. Aquatic vegetation indices and their related sensitivity analysis in reflectance spectra have recently been reported [38]. However, the model used in this work for aquatic vegetation is a terrestrial vegetation model applied in an aquatic context. The impacts of some parameters such as water depth and concentrations of water constituents, which should be of distinct importance especially for submerged vegetation, are not discussed. A systematic sensitivity analysis of the reflectance spectra of various types of aquatic vegetation should be helpful for developing practical aquatic vegetation indices and for retrieving biophysical or biochemical parameters.

The goal of this work is to determine which parameters of the coupled macrophyte-water system can be retrieved from remote sensing measurements and under what conditions. This would help in developing or refining inversion algorithms for aquatic vegetation or optically-complex waters. In addition, by identifying variables of only minor influence, models can be greatly simplified, which facilitates inversion applications [39]. The EFAST method proposed by Saltelli et al. [27] will be utilized to analyze the global sensitivity of vegetation, water parameters and boundary conditions, as this method achieves both accuracy and efficiency. The AVRT model will be utilized to simulate a large data set, which takes the variation of different parameters into consideration, and is analyzed via EFAST. It should be emphasised that the sensitivity analysis in this study is performed on the simulation data (no field measured data used), since most of parameters considered are not controllable in field measurement, which brings difficulties to the sensitivity analysis. In this paper, the global sensitivity analysis includes the following two parts: (1) to analyze 
the sensitivity of AVRT model input parameters to canopy spectra in several cases which are set by subdividing the range of water depth and LAI; and (2) to analyze the sensitivity of input parameters to conventional terrestrial vegetation indices NDVI, SAVI, and aquatic vegetation indices NDAVI and SAVI in several cases, because these broadband vegetation indices are widely used and perform well in applications of multispectral satellite data, which is an important data source in wetland remote sensing. Unlike the use of ocean-color satellite data in deep waters for studying phytoplankton concentration, which relies on spectral signatures in the visible domain, detection of vegetation in shallow-waters requires that we use spectral signatures in both visible and near infrared domains. The spectral response functions of Sentinel-2A can be used to calculate the band equivalent reflectance and then to obtain the vegetation index mentioned above. Because the optical response of floating vegetation is very similar to that of emergent vegetation, and because emergent vegetation is more representative, the targets in this paper are emergent vegetation and submerged vegetation.

The rest of the paper is organized as follows: in Section 2 the AVRT model and the EFAST method used in the paper are briefly introduced. Section 3 shows the ranges and distributions of model input parameters and introduces the spectral advantages of Sentinel-2A for monitoring aquatic vegetation in shallow water bodies, and explains the motivation for selecting Sentinel-2A as the reference sensor. In Section 4, the results of the two parts mentioned above are presented. Section 5 states the comprehensively discussion about these results. Finally, Section 6 concludes this paper and suggests directions for future research.

\section{Methodology}

\subsection{AVRT Model}

To discuss adequately the interactions between water columns and vegetation while maintaining a balance between accuracy and efficiency, we adopt some basic assumptions in the AVRT model: (1) the whole system can be divided into several layers, in vertical direction, whose optical properties are homogeneous. A single layer can be water, vegetation or the water-vegetation mixed medium; (2) the thickness of the water surface is taken as zero; (3) the bottom is assumed to be Lambertian.

The type of medium in each layer is determined mainly by the water depth, plant height, LAI and the volume fraction of water. If the water depth is greater than the plant height, submerged vegetation is taken as the object and each layer in the system is considered, partly or totally, to contain water, which means it could be water or the mixed medium. Otherwise, the system could be emergent vegetation or floating vegetation, and each layer could be vegetation or the mixed medium. Furthermore, if LAI of a certain layer is larger than the threshold, it is determined that this layer must contain vegetation. And if this layer has been judged to contain partly water, it is determined to be the mixed medium. It is logically similar for the volume fraction of water when deciding whether the layer is water or mixed medium.

The entire system also includes the air-water interface and the bottom. After deciding each layer type, the scattering matrix, which includes the reflectance and transmittance, is calculated according to the optical properties of the medium. In the AVRT model, PROSEPCT-5 [40] is utilized to calculate the reflectance and transmittance of a single leaf, and after that it is used in the modified SAILH model to calculate the scattering coefficients of vegetation. The scattering and absorption characteristics of the water column are determined by bio-optical models [41-46]. The Cox-Munk [47] model is utilized to calculate the surface scattering matrix. The detailed calculation process is presented in Zhou et al. [26]. The reflectance matrix of the whole system is calculated using an iterative adding method, which refers to Cooper et al. [48] and Verhoef [49]. Considering both direct and diffuse incident radiations, the canopy bidirectional reflectance can be obtained.

In this paper, the AVRT model is utilized to simulate the canopy spectra of emergent vegetation and submerged vegetation. Apart from the bottom and water surface, the whole system is divided for simplicity into two layers. The lower layer is the water-vegetation mixed medium for both emergent 
vegetation and submerged vegetation. The upper layer for emergent vegetation is the vegetation medium, and that for submerged vegetation is the water medium. The input parameters required in the simulation will be introduced in Section 3.1.

\subsection{EFAST Method}

This section will introduce the EFAST method. It is the extended version of FAST method. When analyzing the model defined as $y=f\left(x_{1}, x_{2}, \ldots, x_{n}\right)$ in the FAST method, a curve is defined as Equation (1) to explore the $n$-dimensional space of input parameters [27]:

$$
x_{i}(s)=G_{i}\left(\sin \omega_{i} s\right), \forall i=1,2, \ldots, n,
$$

where $x_{i}$ stands for the $i$-th input parameter, $s$ is an independent variable, $G_{i}$ is the transformation function and $\omega_{i}$ is a frequency associated with each input parameter. As $s$ varies, all the input parameters change along the curve simultaneously in $n$-dimensional space. Every input oscillates periodically at its corresponding frequency $\omega_{i}$. If $x_{i}$ has significant impact on the model output $y$, it should have high amplitude at its frequency $\omega_{i}$. After Fourier expansion, the model $f\left(x_{1}(s), x_{2}(s), \ldots, x_{n}(s)\right)=f(s)$ can be expressed as Equation (2):

$$
f(s)=\sum_{j=-\infty}^{+\infty}\left(A_{j} \cos j s+B_{j} \sin j s\right), j \in Z,
$$

where $A_{j}$ and $B_{j}$ are the Fourier coefficients. The spectrum of the Fourier expansion was defined as $\Lambda_{j}=A_{j}{ }^{2}+B_{j}{ }^{2}$. Because $f(s)$ was a real-valued function, they are symmetrical and could be expressed as: $A_{-j}=A_{j}, B_{-j}=-B_{j}, \Lambda_{-j}=\Lambda_{j}$. Therefore, the output variance $D_{i}$ arising from $x_{i}$ and the total variance $D$ could be estimated by adding the spectra:

$$
\begin{gathered}
D_{i}=\sum_{p \in Z^{0}} \Lambda_{p \omega_{i}}=2 \sum_{p=1}^{+\infty} \Lambda_{p \omega_{i}}, \\
D=\sum_{j \in Z^{0}} \Lambda_{j}=2 \sum_{j=1}^{+\infty} \Lambda_{j},
\end{gathered}
$$

where $Z^{0}$ means all integers except the 0 . The first order sensitivity index $S_{i}$ of $x_{i}$ could be:

$$
S_{i}=\frac{D_{i}}{D}
$$

After that, Saltelli et al. [27] combined the idea of the variance decomposition method with that of the FAST method. The total output variance would be decomposed into the variance associated with each input or the interactions among the input parameters.

To determine the total sensitivity index $S_{T i}$ of $x_{i}$, the frequency of $x_{i}$ was set to $\omega_{i}$ while the frequency of the rest of input parameters was $\omega_{-i}$, which is different from. The symbol $-i$ refers to the complementary set of $i$. After calculating the spectrum at the frequency $\omega_{-i}$ and higher harmonics $p \omega_{-i}$, the partial variance $D_{(-i)}$ could be obtained just as in Equation (4). It means that the variance unrelated to $x_{i}$, then the variance $D_{T i}$ which is the complementary set of $D_{(-\mathrm{i})}$ could be calculated by subtracting $D_{(-\mathrm{i})}$ from $D . S_{T i}$ could be defined as:

$$
S_{T i}=\frac{D-D_{(-i)}}{D} .
$$

The total sensitivity index $S_{T i}$ describes the impact of $x_{i}$ and the interactions between $x_{i}$ and the rest of input parameters on the model output. It is more comprehensive than the first order sensitivity 
index. Obviously, the sum of first order sensitivity indices of all input parameters should be less than 1 whereas the sum of total order sensitivity indices will be greater than 1 . This sum would equal 1 only if all the interaction items were zero.

The calculation process of EFAST is introduced in detail in Appendix A. In this paper, we will follow the process to make the global sensitivity analysis. The ranges and distributions of all the input parameters of AVRT will be introduced in Section 3.1.

\section{Data}

In this section, the variability ranges and distributions of the input parameters are introduced. They are significant to sampling. After sampling, the input data set is entered into the AVRT model to simulate the canopy spectra of emergent vegetation and submerged vegetation in the range of 400-1000 nm, the global sensitivity analysis (abbreviated as GSA) is performed for each wavelength to obtain the high sensitivity band ranges of each parameter. Next a description of Sentinel-2A satellite is given, including the band settings and the spectral response functions, which are used to calculate first the band equivalent reflectance and then the vegetation index.

\subsection{Input Parameters}

A systematic exploration of the parameter space is necessary for GSA. It is important to select an approximate and representative sample set, because the macrophyte-water coupled system is too complicated to test all possible combinations of parameter values. Efficient sampling of the parameter space requires some priori knowledge of the probability distribution of parameter values. This ensures that unlikely or implausible parameter values and unlikely combinations are not given equal weight to the more likely ones [36]. The input parameters of AVRT could be divided into vegetation parameters, water column parameters and the boundary conditions. The ranges and descriptions of the input parameters are given in Table 1. The ranges of leaf biophysical parameters refer to the technical documents of PROSEPCT-5. The ranges of water column parameters refer to the field measurements reported in relevant papers (such as Cchla [43] and SPM [50]), and the ranges set by the bio-optical models (Btsm [46] and aCDOM [51]).

Due to the lack of priori knowledge, the probability distributions of all parameters are set to uniform, that is all parameters are equally likely to be found anywhere in their range of variation. The results of the GSA could change when the range of variation of the parameters changed. Therefore, it is of great importance to establish several cases by combining different parameter ranges, which could also reveal the sensitive conditions for each parameter. In this paper, the water depth is divided into shallow (0.1-1.0, unit: $\mathrm{m}$ ) and deep (1.0-2.0, unit: $\mathrm{m}$ ) conditions, which reflect the strength of the attenuation caused by water columns. The range of LAI is set to sparse ( $0-2$, dimensionless) and dense (2-4, dimensionless) to represent the extent of vegetation cover. After a couple of combinations, four cases can be obtained: shallow-sparse, shallow-dense, deep-sparse and deep-dense. The observation conditions are shown at the end of Table 1.

As mentioned in Section 2.1, the system is divided into two layers for emergent vegetation and submerged vegetation. So, the definitions of $\mathrm{Hw}$ and $\mathrm{Hp}$ in Table 1 are actually the height of a single layer in the system. A detailed schematic of vertical structures for the two kinds of vegetation is shown as Figure 1. In Figure 1a, Hw is the height from bottom to the water surface, that is, the actual water depth, which represents the height of the water-vegetation mixed medium. Hp is the height from the water surface to the top of the canopy, which represents the height of the layer totally full of vegetation. As for submerged vegetation, $\mathrm{Hp}$ is the height from bottom to the top of the canopy, that is, the actual plant height, which represents the height of the mixed medium. Hw is the height from the top of the canopy to the water surface, which represents the height of the water layer. 
Table 1. The descriptions and ranges of input parameters. The observation conditions are indicated at the end of Table 1, which include solar and viewing zenith angle and the relative azimuth angle in air.

\begin{tabular}{|c|c|c|c|}
\hline Parameter & Description & Unit & Range \\
\hline \multirow{2}{*}{$\mathrm{Hw}^{1}$} & \multirow{2}{*}{$\begin{array}{l}\text { Water depth for emergent vegetation } \\
\text { The height of the upper water layer for submerged vegetation }\end{array}$} & \multirow{2}{*}{$\mathrm{m}$} & 0.1-1.0 (Shallow) \\
\hline & & & 1.0-2.0 (Deep) \\
\hline Hр & $\begin{array}{l}\text { The height of the upper vegetation layer for emergent vegetation } \\
\text { Plant height for submerged vegetation }\end{array}$ & $\mathrm{m}$ & $0.1-1.0$ \\
\hline $\mathrm{N}$ & Leaf structure parameter & - & $1-2.5$ \\
\hline $\mathrm{Cab}$ & Concentration of chlorophyll $\mathrm{a}+\mathrm{b}$, in leaves & $\mu \mathrm{g} / \mathrm{cm}^{2}$ & $10-80$ \\
\hline Car & Concentration of carotenoid, in leaves & $\mu \mathrm{g} / \mathrm{cm}^{2}$ & $10-30$ \\
\hline $\mathrm{Cw}$ & Concentration of equivalent water thickness, in leaves & $\mu \mathrm{g} / \mathrm{cm}^{2}$ & $0.004-0.04$ \\
\hline $\mathrm{Cm}$ & Concentration of dry matter, in leaves & $\mu \mathrm{g} / \mathrm{cm}^{2}$ & $0.002-0.01$ \\
\hline \multirow{2}{*}{ LAI } & \multirow{2}{*}{ Leaf area index } & \multirow{2}{*}{-} & 0-2 (Sparse) \\
\hline & & & 2-4 (Dense) \\
\hline LIDFa & $\begin{array}{l}\text { Leaf inclination distribution function parameter a } \\
\text { (which represents the average leaf slope) }\end{array}$ & - & $-1-1$ \\
\hline LIDFb & $\begin{array}{l}\text { Leaf inclination distribution function parameter } b \\
\text { (which represents the distribution's bimodality) }\end{array}$ & - & $-1-1$ \\
\hline $\mathrm{Fw}$ & volume fraction of water in a layer & - & $0.7-1$ \\
\hline Cchla & Concentration of chlorophyll a, in water & $\mathrm{mg} / \mathrm{m}^{3}$ & $0-80$ \\
\hline Btsm & Coefficient to calculate scattering of total suspended matter & - & $1-5$ \\
\hline SPM & Concentration of suspended matter, in water & $\mathrm{g} / \mathrm{m}^{3}$ & $0-80$ \\
\hline $\mathrm{aCDOM}$ & Absorption coefficient of CDOM at $375 \mathrm{~nm}$ & $/ \mathrm{m}$ & $0.5-3$ \\
\hline Rbtm & Reflectance of bottom & - & $0-1$ \\
\hline SZA & Sun zenith angle in air & degree & 30 \\
\hline VZA & Viewing zenith angle in air & degree & 0 \\
\hline RA & Relative azimuth angle in air & degree & 0 \\
\hline
\end{tabular}

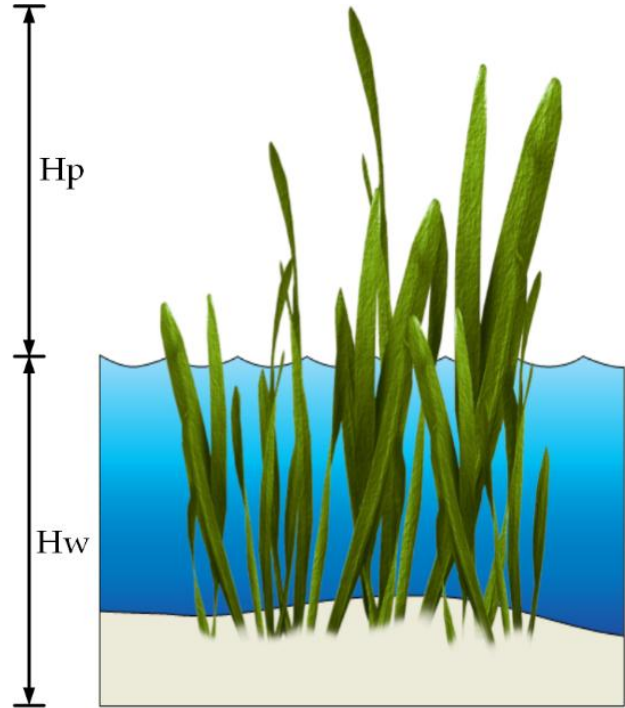

(a)

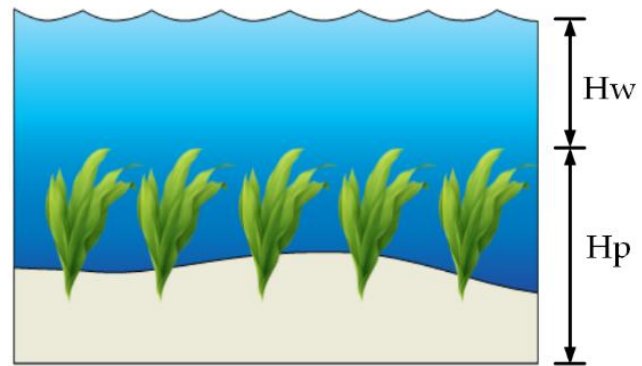

(b)

Figure 1. The Schematic of vertical structures for emergent vegetation and submerged vegetation.

(a) Emergent vegetation; (b) Submerged vegetation. 
The setting of sample size should take the computational efficiency and robustness into consideration. The robustness of GSA means that the sensitivity index does not change drastically with the increase of sample size. In this study, the results of different sample sizes have been compared in advance, and finally it was set to $16 \times 513=8208$, where 16 represents the number of model input parameters, and 513 is the number of samples required for a single input parameter. Each sample contains the input parameters needed for the model, and the corresponding canopy reflectance can be obtained after calculation. After GSA, the curve of the sensitivity index is obtained for each parameter.

\subsection{Sentinel-2A}

The goal in our sensitivity analysis is to obtain wavelength relationships that maximize the possibility of retrieving some important parameters in wetland aquatic vegetation areas. These wavelength relationships were compared with the available bands on the broadband sensors such as Sentinel-2A. Macrophytes have complex spatial mosaic structures and, therefore, require concurrent high spatial and spectral resolution remote sensing data. The Sentinel-2A is a twin polar-orbiting satellite mission with wide swath, high spatial and spectral resolution and revisiting frequency that is expected to have significant potential for mapping aquatic vegetation in complex wetland ecosystems [52]. Furthermore, the Multispectral Imager Instrument (MSI) carried on Sentinel-2A has 13 spectral bands, especially four bands that cover the "red edge" region, which is in the range of 700-750 $\mathrm{nm}$. These bands can provide enriched capabilities for the detection of aquatic vegetation. The open access and free-of-charge policy adopted for the Sentinel-2A data products favor routine environmental applications (including wetlands). Therefore, Sentinel-2A is chosen as the reference sensor in this paper, which aims to provide theoretical support for future retrieval of aquatic vegetation parameters in the context of optical remote sensing.

The MSI carried by Sentinel-2A has spectral bands in visible, near infrared and shortwave infrared region, with spatial resolution of $10 \mathrm{~m}, 20 \mathrm{~m}$ and $60 \mathrm{~m}$. There are four bands at $10 \mathrm{~m}: 490 \mathrm{~nm}(\mathrm{~B} 2)$, $560 \mathrm{~nm}$ (B3), $665 \mathrm{~nm}$ (B4), $842 \mathrm{~nm}$ (B8); six bands at $20 \mathrm{~m}: 705 \mathrm{~nm}$ (B5), $740 \mathrm{~nm}$ (B6), $783 \mathrm{~nm}$ (B7), $865 \mathrm{~nm}$ (B8a), $1610 \mathrm{~nm}$ (B11), $2190 \mathrm{~nm}$ (B12); three bands at $60 \mathrm{~m}: 443 \mathrm{~nm}$ (B1), $945 \mathrm{~nm}$ (B9) and $1375 \mathrm{~nm}$ (B10). The spectral response functions of Sentinel-2A are used to calculate the band equivalent reflectance by convolving with the simulation data, and then to calculate the broadband vegetation index analyzed in the study. Figure 2 is the plot of the spectral response functions of bands (B1-B9) in 400-1000 nm. In Section 4.2, a brief description of these vegetation indices is presented.

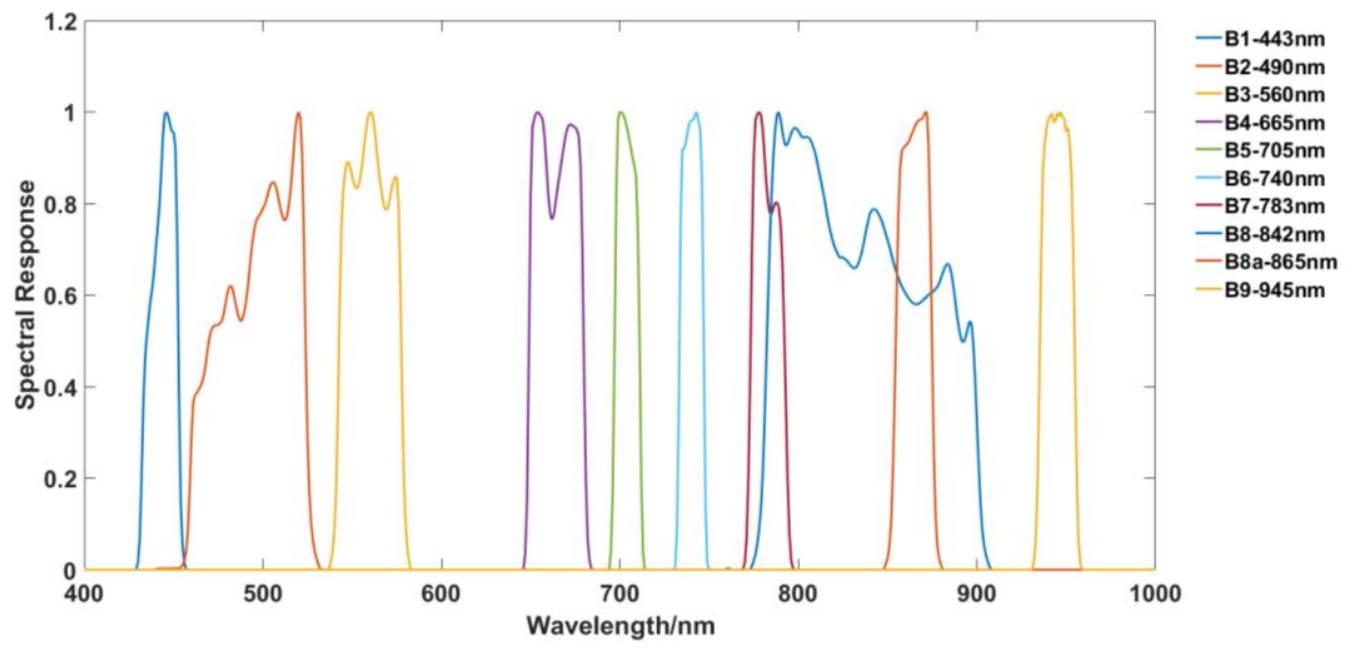

Figure 2. The Sentinel-2A spectral response functions of bands (B1-B9) in 400-1000 nm. 


\section{Results}

\subsection{GSA to Reflectance in Different Cases}

For the cases set in Section 3.1, the total order sensitivity indices of input parameters are calculated wavelength by wavelength. It can be assumed that if a parameter is highly sensitive over a band range, it will have a significant effect on canopy reflectance over this range, and inversion of the parameter by reflectance in this band range should be possible.

To show clearly the sensitivity of different input parameters at each wavelength, the total order sensitivity indices of all parameters are normalized to the range $0-1$, which refers to the contribution of a single parameter to the sum of all parameter's sensitivity. To evaluate the impact of a single parameter on the reflectance over the whole band range $(400-1000 \mathrm{~nm})$, the influence of the parameter $(P I)$ is defined as the ratio of the sum of the normalized total order sensitivity (NST) index of the parameter to the sum of all the parameters' NST over the entire band range:

$$
P I_{i}=\frac{\sum_{\lambda=400}^{1000} N S T_{i}(\lambda)}{\sum_{i=1}^{16} \sum_{\lambda=400}^{1000} N S T_{i}(\lambda)}
$$

In Equation (7), $P I_{i}$ stands for the impact of the $i$-th input parameter, and $\lambda$ represents the wavelength, which is in the range of $400-1000 \mathrm{~nm}$. PI means the NST proportion of the $i$-th parameter in all parameters over the whole band range. Next, the analysis results of emergent vegetation and submerged vegetation will be introduced.

\subsubsection{Emergent Vegetation}

Figure 3 is the cumulative plot of NST for emergent vegetation in four different cases. The larger area of a parameter in the band range means it is more significant to the reflectance.
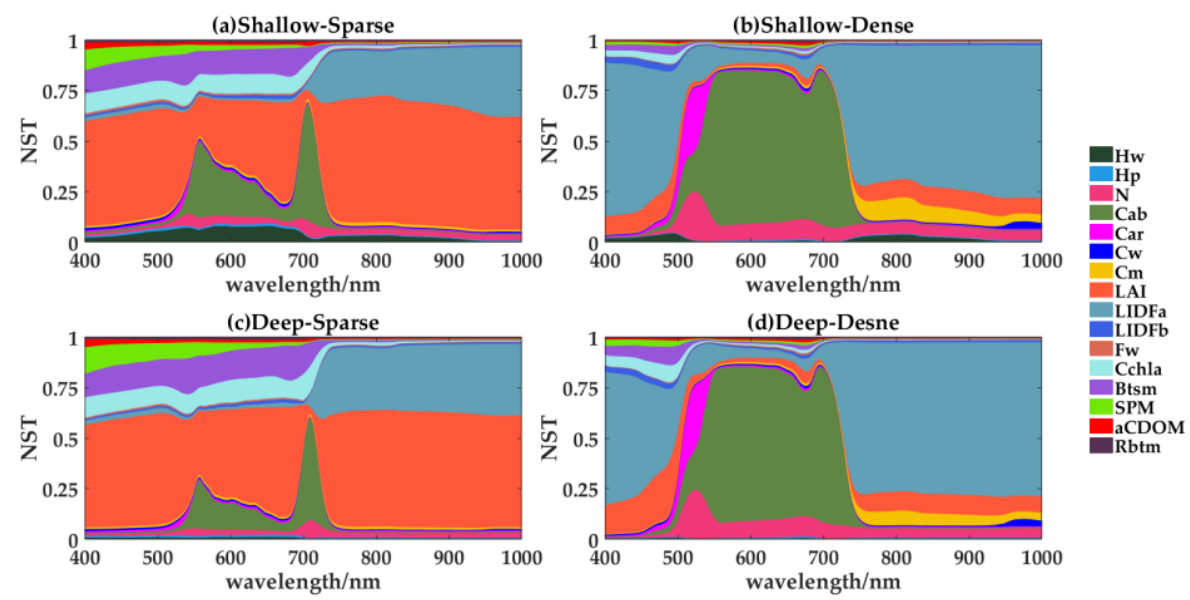

Figure 3. The cumulative plot of normalized total order sensitivity (NST) for emergent vegetation in four different cases. (a), (b), (c), (d) represent the cases of shallow-sparse, shallow-dense, deep-sparse and deep-dense, respectively. The area enclosed by the curve and the horizontal axis (the first parameter in the list, $\mathrm{Hw}$ ), and between the curves (except $\mathrm{Hw}$ ) represents the value of NST.

Combined with Equation (7), high sensitivity vegetation parameters include N, Cab, Car, Cm, LAI and LIDFa. High sensitivity water parameters include Hp, Cchla, Btsm and SPM. It can be seen from Figure 3 that the sensitivity of vegetation parameters is far greater than that of the water parameters for all four cases defined in this study, especially Cab, LAI and LIDFa. The water parameters score only 
weakly high sensitivity in sparse cases, and the sensitivity is higher in deep-sparse case (Figure 3c) than shallow-sparse case (Figure 3a). Taking Cchla as an example, the values of PI in (a) and (c) are 0.054 and 0.059 respectively.

Figure 4 shows the high sensitivity parameters in different cases varying with wavelength. Comparing with (a), (b), (c) and (d), the range of depth (shallow or deep) has no significant effect on the curves of high sensitivity parameters, and it changes the values of NST only in some regions. The range of LAI (sparse or dense) has an obvious effect on both vegetation and water parameters, which can be partially explained by the vertical structure of emergent vegetation. When emergent vegetation is not too sparse, the water just acts as a background and apparently cannot influence the canopy reflectance.

The high sensitivity vegetation parameters of AVRT can be divided into leaf parameters N, Cab, Car, $\mathrm{Cm}$, and canopy parameters LAI, LIDFa. Figure 4 shows that the sensitivity values of leaf parameters and LIDFa are much greater in dense cases than in sparse cases whereas the sensitivity of LAI is the opposite. In sparse cases, the sensitivity values of $\mathrm{N}$, Car and $\mathrm{Cm}$ are almost negligible. In dense cases, the high sensitivity regions of $\mathrm{N}$ and Car are around $510 \mathrm{~nm}$ and $500 \mathrm{~nm}$ respectively. The region at $500 \mathrm{~nm}$ corresponds to the absorption band of Car. The absorption spectrum of Car is also shown in Figure 4. The high sensitive range of $\mathrm{Cm}$ is mainly around 700-900 $\mathrm{nm}$ where the maximum is at $810 \mathrm{~nm}$. The sensitivity of $\mathrm{Cm}$ is slightly lower in case (d) than (b) where the maximum of NST are 0.073 and 0.111 respectively.

The sensitivity of Cab is very high in the visible region. In sparse cases, the NST values of Cab peak are at $550 \mathrm{~nm}$ and $710 \mathrm{~nm}$ whereas the trough is at $670 \mathrm{~nm}$. The values in shallow cases are slightly higher than those in deep cases, where the maxima are 0.577 and 0.505 in (a) and (c), respectively. In dense cases, the sensitivity of Cab is very high in the range of 550-750 nm while the trough near $670 \mathrm{~nm}$ is no longer obvious. The values of NST within this range are kept at about 0.755 , which shows negligible response to the change in water depth. In general, it can be summarized from the above analysis that the greatest sensitivity to Cab exists in three characteristic bands located at $550 \mathrm{~nm}$, $670 \mathrm{~nm}$ and $710 \mathrm{~nm}$. The absorption spectrum of Cab is also shown in Figure 4, where it can be seen that the trough is located at $550 \mathrm{~nm}$ while the peak is at $670 \mathrm{~nm}$. Besides, the "red edge" position of the vegetation canopy spectrum is located at $710 \mathrm{~nm}$. Thus, the optical properties of chemical substances in the leaves, such as Car and Cab, show up well in GSA.

LAI and Leaf Angle Distribution (LAD) are the two significant parameters that mainly control the amount of light interception and dominate the canopy reflectance for terrestrial vegetation in the visible and NIR regions. LAI can be treated as an important factor for assessing the growth status and biomass. LIDFa and LIDFb are adopted to describe LAD in AVRT model. The former controls the average leaf slope and the latter controls the bimodality distribution of leaves. According to Figure 4, the sensitivity values of LAI and LIDFa are strongly affected by the extent of vegetation cover. In sparse cases, the NST values of LAI are very significant within the range of 400-500 nm, 650-680 nm and $730-1000 \mathrm{~nm}$ whereas the troughs are around $550 \mathrm{~nm}$ and $710 \mathrm{~nm}$. It can be considered that the NST values of LAI and Cab are negatively correlated. The maximum NST of LAI is about $0.5-0.6$ in (a) and (c), which is almost unaffected by the variation of water depth. The range of influence of LIDFa is $730-1000 \mathrm{~nm}$, and the maximum of NST is about $0.3-0.4$ in (a) and (c). The value of NST is close to zero in the visible range. In dense cases, the sensitivity of LAI declines abruptly to around $0.1-0.2$, especially in 400-500 $\mathrm{nm}$ and 650-680 $\mathrm{nm}$. On the contrary, the sensitivity of LIDFa increases strongly, especially in $400-500 \mathrm{~nm}$ and 730-1000 nm with a maximum NST of $0.765 \mathrm{in}(\mathrm{b})$ and $0.755 \mathrm{in}$ (d). The sensitivity of LIDFa is relatively low near $500-710 \mathrm{~nm}$, due to the high sensitivity of Cab in this range.

The sensitivity values of water parameters in dense cases are almost negligible. In sparse cases, the sensitive ranges of Cchla and Btsm are mainly in the visible band of 400-700 nm, and the range of influence of SPM is mainly in 400-500 nm. Their maxima values of NST are all around 0.1-0.2. 


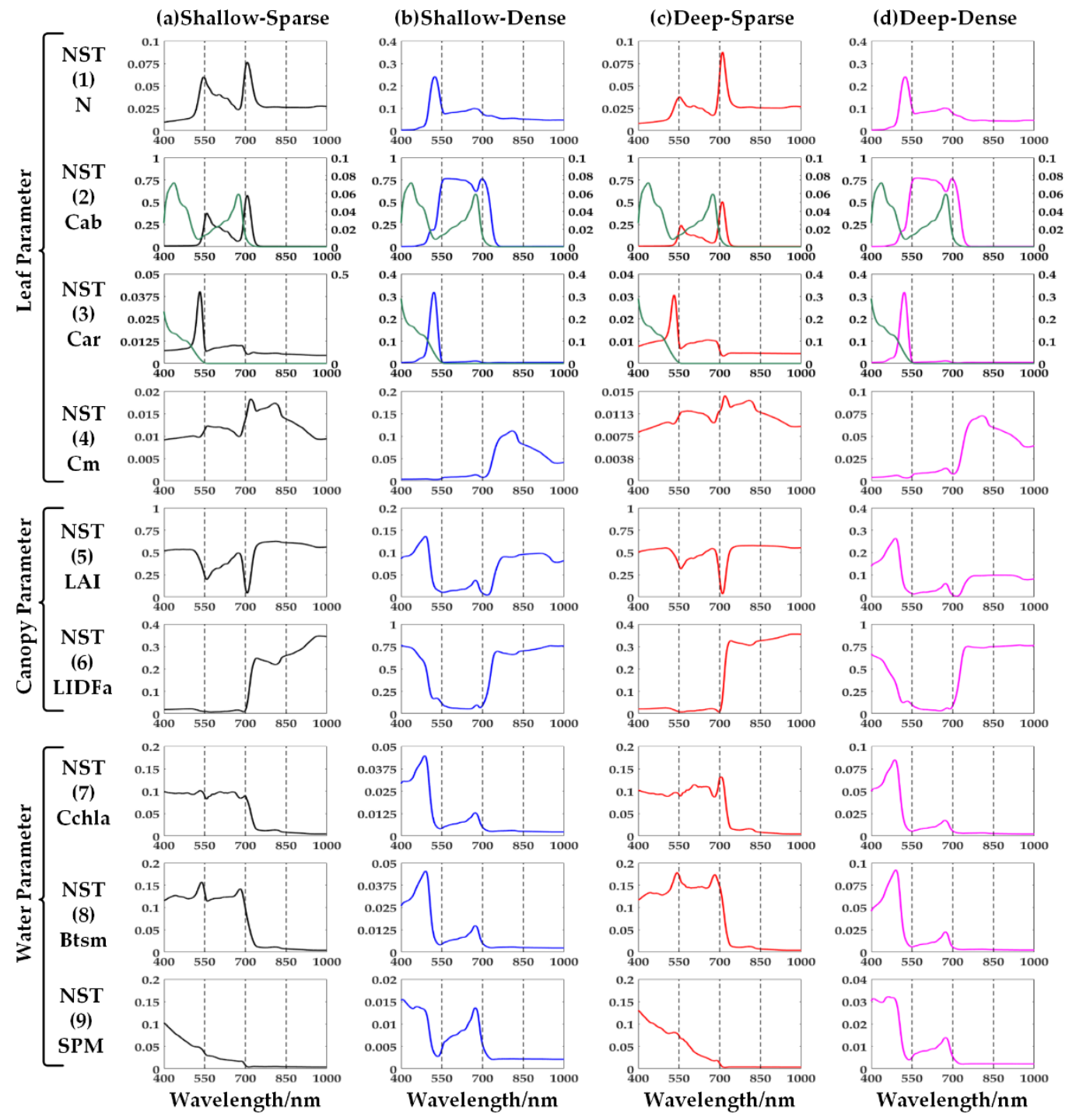

Figure 4. In four different cases, the curves of high sensitivity parameters varying with wavelength. The columns (a), (b), (c), (d) represent the case of shallow-sparse, shallow-dense, deep-sparse and deep-dense, respectively. (1)-(9) represent the high sensitivity parameters, (1): N, (2): Concentration of chlorophyll $\mathrm{a}+\mathrm{b}$ (Cab), (3): Concentration of carotenoid (Car), (4): Concentration of dry matter (Cm), (5): Leaf area index (LAI), (6): Leaf inclination distribution function parameter a (LIDFa), (7): Concentration of chlorophyll a, in water (Cchla), (8): Coefficient to calculate scattering of total suspended matter (Btsm), (9): Concentration of suspended matter (SPM). It should be noted that the curves of absorption coefficient of Cab and Car are also shown in (2) and (3). The vertical axis on the right shows the range of the absorption coefficient.

\subsubsection{Submerged Vegetation}

The GSA results of submerged vegetation are presented next: they are clearly different from the results for emergent vegetation. Figure 5 is the cumulative plot of NST for submerged vegetation for four different cases. According to GSA, the high sensitivity water parameters include Hw, Cchla, Btsm, SPM and aCDOM, and the high sensitivity vegetation parameters include Hp, Cab and LAI.

Unlike emergent vegetation, the sensitivity values of water parameters are far greater than those of vegetation parameters, especially Cchla and Btsm, both of which are highly sensitive over the entire band range (Figure 5). In the case of (a) (shallow-sparse), for example, the PI values of these two parameters are 0.388 and 0.428 , respectively, while the most sensitive vegetation parameter LAI 
scores only 0.012. In deep cases, the effects of vegetation parameters are almost obscured by water parameters, even in deep-dense case. No matter what case, the sensitivity of bottom reflectance is almost negligible. The variation of sensitivity is fully investigated in Figure 6, which shows the curves of high sensitivity parameters in different cases varying with wavelength.
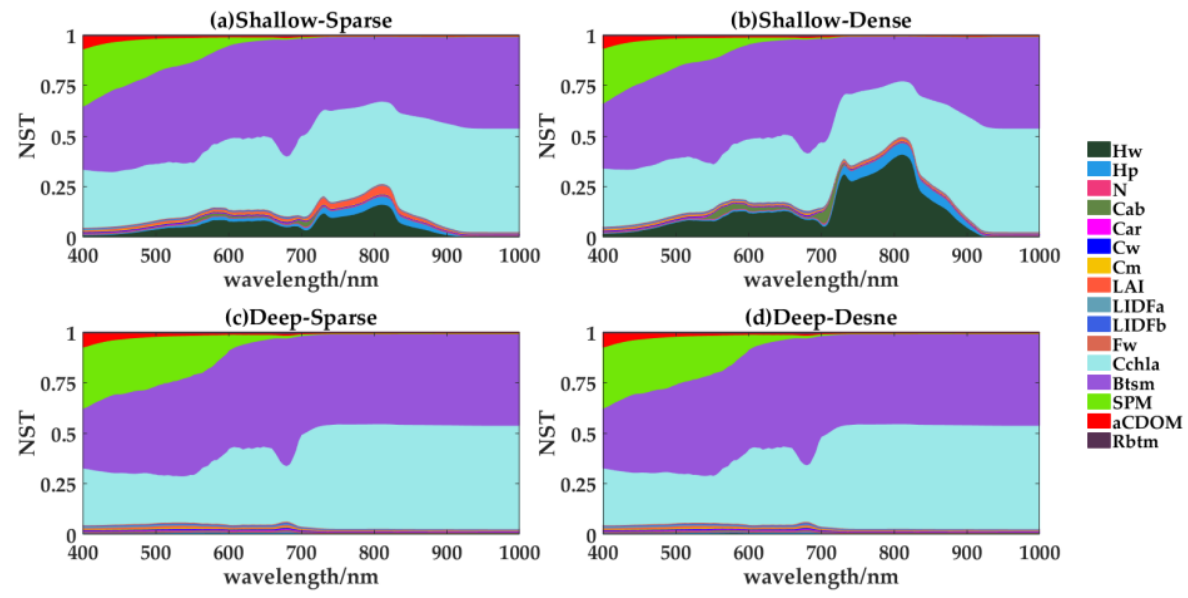

Figure 5. The cumulative plot of NST for submerged vegetation in four different cases. (a), (b), (c), (d) represent the cases of shallow-sparse, shallow-dense, deep-sparse and deep-dense, respectively. The area enclosed by the curve and the horizontal axis (the first parameter in the list, Hw), and between the curves (except $\mathrm{Hw}$ ) represents the value of NST.

The influence ranges of SPM and aCDOM are relatively narrow, with the decreasing trend of NST at 400-600 $\mathrm{nm}$ and 400-550 $\mathrm{nm}$, respectively. The sensitivity values of SPM and aCDOM in deep cases are slightly greater than those in shallow cases. Hw is more sensitive in shallow cases than in deep cases, and the values of $P I$ in these cases are $0.055,0.124,0.003$ and 0.004 respectively, indicating that the influence of $\mathrm{Hw}$ on the canopy reflectance could not be significant when the water depth increases to a certain extent. The curves of Hw have different patterns in shallow and deep cases. In shallow cases, the influence range of $\mathrm{Hw}$ is mainly located in 700-900 nm, while in deep cases it changes to $500-700 \mathrm{~nm}$, the peak being at $600 \mathrm{~nm}$. Hw is more sensitive in dense cases than in sparse cases, indicating that the increase of water depth will make the attenuation more obvious in dense cases than sparse cases.

In either case, Cchla and Btsm have very significant effects over the whole band range. They have different influence ranges in shallow and deep cases. The sensitivity values in deep cases are slightly higher than those in shallow cases. Taking Cchla as an example, $P I$ is $0.388,0.347,0.403$ and 0.404 in these four cases, respectively. In shallow cases, the curve of Cchla has a minor trough around $680 \mathrm{~nm}$ and $810 \mathrm{~nm}$, while the peak of Btsm's curve is near $680 \mathrm{~nm}$. In deep cases, there are similar relationships between Cchla and Btsm, indicating that it would be helpful for constructing the inversion algorithm by using the bands around $680 \mathrm{~nm}$ to suppress the impact of Btsm. Currently, this band has been applied to construct the three-band [53] and four-band [54] semi-analytical method to extract the concentration of chlorophyll in high-turbidity water.

The sensitivity features of vegetation parameters deserve more attention: they can provide the theoretical basis for inversion of submerged vegetation parameters. Relative to shallow cases, the NST values of vegetation parameters are much lower in deep cases, since the increase of water depth means the canopy signal will be attenuated more strongly. The sensitivity values of $\mathrm{Hp}$ are slightly lower than those of LAI and Cab, and the values of PI in four cases are $0.016,0.020,0.004$ and 0.003 , respectively. The curve of $\mathrm{Hp}$ has no stable shape because it does not directly reflect the biophysical-biochemical properties of the plant. It mainly reflects the vertical height of the water-vegetation mixed medium in the model. In general, its influence range is located in 700-900 $\mathrm{nm}$ in shallow cases. 

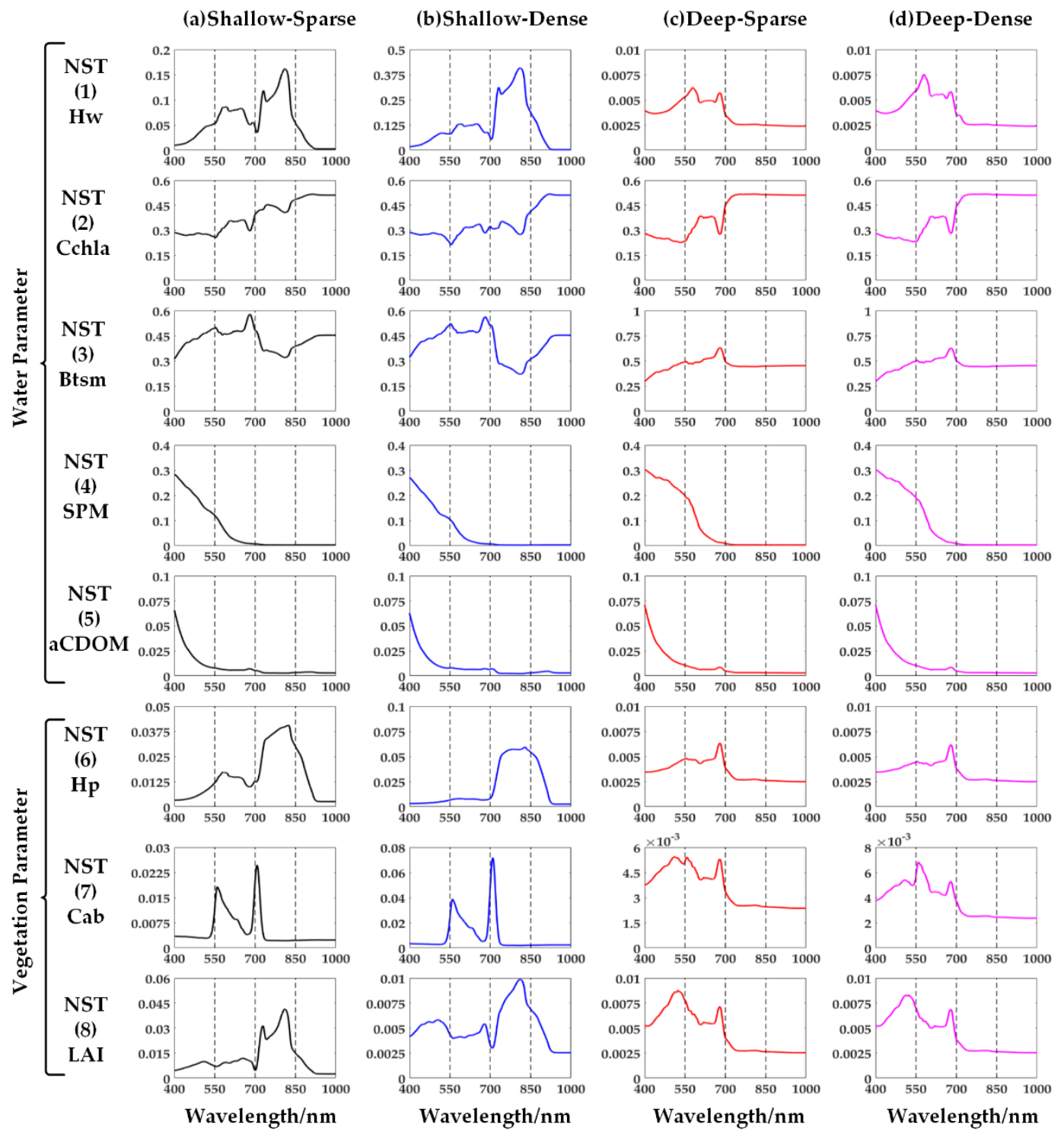

Figure 6. In four different cases, the curves of high sensitivity parameters varying with wavelength. The columns (a), (b), (c), (d) represent the case of shallow-sparse, shallow-dense, deep-sparse and deep-dense, respectively. (1)-(9) represent the high sensitivity parameters of the model, (1): The height of the upper water layer (Hw), (2): Concentration of chlorophyll a, in water (Cchla), (3): Coefficient to calculate scattering of total suspended matter (Btsm), (4): Concentration of suspended matter (SPM), (5): Absorption coefficient of CDOM at $375 \mathrm{~nm}(\mathrm{aCDOM})$, (6): Plant height (Hp), (7): Concentration of chlorophyll $\mathrm{a}+\mathrm{b}(\mathrm{Cab}),(8)$ : Leaf area index (LAI).

The sensitivity patterns of Cab and LAI are stable, especially Cab. The bands around $550 \mathrm{~nm}$ and $710 \mathrm{~nm}$ are high sensitivity regions of $\mathrm{Cab}$, while the other regions have almost no sensitivity, which is similar to the result of 4.1.1. However, the GSA results for LAI are slightly different from the conclusion in 4.1.1. In shallow cases, the influence range of LAI is 700-900 nm while the NST values are much smaller in the shallow-dense case. For deep cases, its influence shifts to around $500 \mathrm{~nm}$, and the NST values drop to the order of $10^{-3}$, which cannot provide useful information. As with the above results, $\mathrm{Cab}$ is more sensitive in dense cases than in sparse cases, while LAI is more sensitive in sparse cases than in dense cases. 
The inversion methods of vegetation parameters are usually based on the vegetation index, which is a common tool for the terrestrial vegetation remote sensing. In the following, the GSA will be performed on the vegetation index.

\subsection{GSA to VIs in Different Cases}

This section will discuss the GSA results for the high sensitivity parameters to vegetation index identified in 4.1. Vegetation index refers to the band combinations of satellite data. Huete et al. pointed out that the purpose of constructing VI is to enhance the vegetation signal while weakening the impact resulting from the background [55], which makes it an effective approach for inversion. Around this goal, many scholars have constructed various forms of vegetation indices, such as NDVI [56], PVI [56], SAVI [57], and EVI [58].

In Section 4.1, many emergent vegetation and submerged vegetation canopy spectra under different input conditions have been simulated. The canopy spectra are convolved with the spectral response functions of Sentinel-2A to obtain the band equivalent reflectance, and then to enable the vegetation index to be calculated. The sensitivity of model input parameters to vegetation indices can be obtained by EFAST. Table 2 summarizes the formulae of vegetation indices to be analyzed. The bands in the formulae are represented by the band numbers in Section 3.2. For example, B2 indicates the second band of Sentinel-2A (blue band). The value of $L$ for both SAVI and WAVI is 0.5. It should be pointed out that the spatial resolution of all the bands selected in the formulae is $10 \mathrm{~m}$. Although there are other bands with narrower band width in the similar band range (such as B6 and B7, which also locate in the near infrared band), the mixing-pixel problems in wetland remote sensing are more complicated, which means bands with high spatial resolution hold more advantages.

Table 2. The formulae of vegetation indices to be analyzed.

\begin{tabular}{ccc}
\hline VI & Description & Formula \\
\hline NDVI & Normalized Difference Vegetation Index & $\frac{\rho(\mathrm{B} 8)-\rho(\mathrm{B} 4)}{\rho(\mathrm{B} 8)+\rho(\mathrm{B} 4)}$ \\
\hline \multirow{2}{*}{ SAVI } & Soil Adjusted Vegetation Index & $(1+L) \frac{\rho(\mathrm{B} 8)-\rho(\mathrm{B} 4)}{\rho(\mathrm{B} 8)+\rho(\mathrm{B} 4)+L}$ \\
\hline \multirow{2}{*}{ NDAVI } & Normalized Difference Aquatic Vegetation Index & $\frac{\rho(\mathrm{B} 8)-\rho(\mathrm{B} 2)}{\rho(\mathrm{B} 8)+\rho(\mathrm{B} 2)}$ \\
\hline \multirow{2}{*}{ WAVI } & Water Adjusted Vegetation Index & $(1+L) \frac{\rho(\mathrm{B} 8)-\rho(\mathrm{B} 2)}{\rho(\mathrm{B} 8)+\rho(\mathrm{B} 2)+L}$ \\
\hline
\end{tabular}

\subsubsection{Emergent Vegetation}

Figure 7 is the column chart of NST values to vegetation indices mentioned above in four different cases for emergent vegetation. The high sensitivity parameters presented in the chart are the same as those in 4.1.1. The analysis results shown in Figure 7 are similar to those shown in Figure 4 . In sparse cases, LAI is the most sensitive parameter to all vegetation indices, indicating that it is possible to retrieve LAI in sparse cases rather than dense cases via the vegetation index. Whereas the sensitivity of LAI in dense cases is weakened, just like that in Figure 4. The sensitivity of leaf parameters such as $\mathrm{Cab}$ and Car, and LIDFa rises strongly. In case (a) the sensitivity of LAI to NDAVI is greater than to the other indices, and the values of NST are $0.572,0.805,0.893$ and 0.830 respectively. It can be attributed to the fact that the formulae of those indices all contain the near infrared (B8) band, which is the sensitive band of LAI in sparse cases according to Figure 4. However, NDAVI and WAVI, which are formulated by the blue band (B2), perform better than NDVI and SAVI formulated by the red band (B4) because LAI is more sensitive in the blue band than that in the red band. In fact, except for case (c), the NST of LAI to NDAVI is higher than to the other indices, which indicates that for emergent vegetation, NDAVI is more suitable for LAI inversion than the other indices except the deep-sparse case. This result is consistent with the conclusion of Villa et al. that for aquatic vegetation LAI is more sensitive to NDAVI than to the conventional terrestrial vegetation index [38]. In case (c), LAI scores higher sensitivity to 
NDVI, indicating that NDVI is a better option in deep-sparse case. The sensitivity of LAI to NDAVI in (c) is the lowest, probably due to the combined effect of increased water depth and water parameters in the blue band.
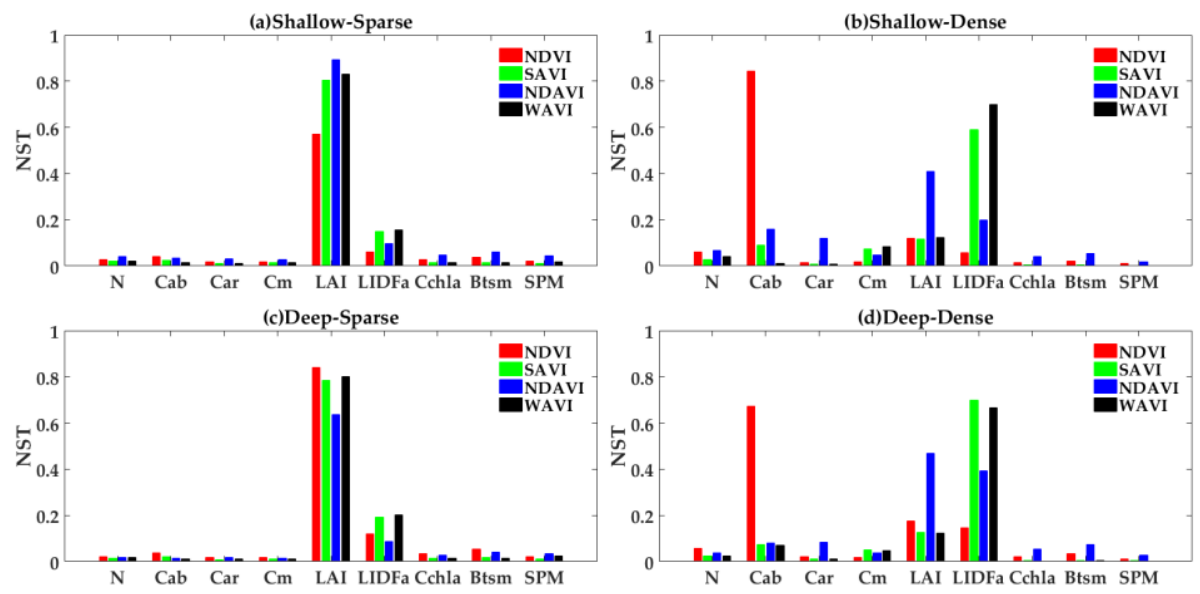

Figure 7. The NST of high sensitivity parameters to vegetation indices for emergent vegetation. (a), (b), (c), (d) represent the case of shallow-sparse, shallow-dense, deep-sparse and deep-dense, respectively.

In dense cases, the sensitivity of leaf parameters increases clearly, especially for Cab. The sensitivity of Cab to NDVI is much higher than that to the other indices. In case (b), the NST of Cab is $0.842,0.090,0.158$ and 0.010, respectively, since NDVI is constructed by the red and near infrared bands, which are the trough and peak respectively in Figure 4, and both of these bands are characteristic. Car is more sensitive to NDAVI than to the other indices due to the high sensitivity in the blue band. As for LIDFa, its sensitivity to the adjusted indices (WAVI and SAVI) is much higher than that to the normalized indices (NDAVI and WAVI), which are constructed by the same band combinations. Taking case (b) as an example, the NST of LIDFa are 0.059, 0.590, 0.199 and 0.700, respectively. This could be a result of the adjustment factor $L$.

There is almost no sensitivity of the water parameters to the vegetation indices, which is plausible since the vegetation index is designed to enhance the contribution of vegetation properties and canopy structural variations, at the same time reducing background (soil and water) effects.

\subsubsection{Submerged Vegetation}

The following are the GSA results for submerged vegetation. Figure 8 is the column chart of NST values to vegetation indices in four above-defined cases. In shallow-sparse case, all the water parameters are sensitive to one or several vegetation indices. Taking SPM as an example, it is sensitive to NDAVI and WAVI. In shallow-dense case, $\mathrm{Hw}$ is the most sensitive parameter to all indices, since all the indices have near infrared bands, where the sensitivity of $\mathrm{Hw}$ is much higher than the other parameters according to Figure 5. The other water parameters are less sensitive to vegetation indices, which could be explained by the lower sensitivity to reflectance in the near infrared region. Among the vegetation parameters, the sensitivities of $\mathrm{Hp}$ and $\mathrm{Cab}$ are insignificant, whereas LAI is sensitive only to the four indices in case (a), to some degree, which is consistent with the results in Figure 5. In case (a), the sensitivity of LAI to NDAVI is higher than to the other indices, and the NST values of LAI to all four indices are $0.115,0.066,0.141$ and 0.049 , respectively, which is similar to the results of Villa et al. as well [38]. 

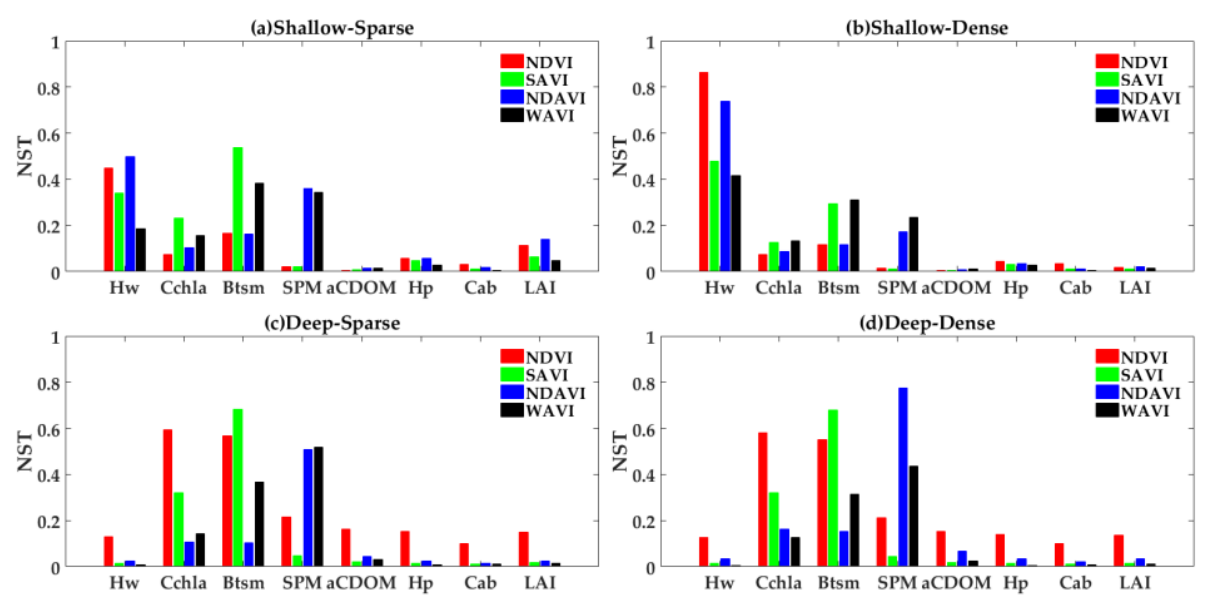

Figure 8. The NST of high sensitivity parameters to vegetation indices for submerged vegetation. (a), (b), (c), (d) represent the cases of shallow-sparse, shallow-dense, deep-sparse and deep-dense, respectively.

However, in deep cases Hw is sensitive only to NDVI while the sensitivity values of the other water parameters rise substantially. SPM is highly sensitive to NDAVI and to WAVI since they both utilize the blue band, which is exactly in the high sensitivity range of SPM. Btsm shows higher sensitivity to SAVI and WAVI than to NDVI and NDAVI, probably due to the adjustment factor $L$. In fact, $\mathrm{Hw}$ is less sensitive to the adjusted vegetation indices in four cases, which means $L$ does effectively suppress the influence of water depth, but it seems that $L$ cannot suppress the effects of water constituent parameters, especially Cchla and Btsm. Considering that the sensitivity values of these two parameters to reflectance over the whole band range are far too significant, especially in deep cases, it is difficult to find the suitable bands to resist the sensitivity of Cchla and Btsm. In cases (c) and (d), although the NST values of vegetation parameters are too low, it should still be pointed out that the sensitivity values of LAI and Cab to NDVI are much higher than to the other indices. Taking (c) as an example, the NST of LAI to the indices are $0.151,0.017,0.023$ and 0.016 , respectively. As for Cab, the values are $0.100,0.012,0.014$ and 0.010 , respectively. These results may provide clues for species identification and vegetation parameters inversion for submerged vegetation.

\section{Discussion}

According to the results in Section 4, the influential variables of the coupled macrophyte-water system, and the conditions under which they are sensitive, can be revealed, given a priori knowledge in the inversion of parameters. For example, it can be seen from Figure 4 that LAI scores high sensitivity around $650-680 \mathrm{~nm}$ and 730-1000 $\mathrm{nm}$ for emergent vegetation in sparse cases. These two band ranges have been utilized to build many vegetation indices such as NDVI and SAVI, which have greatly helped retrieval of LAI for terrestrial vegetation. Therefore, the high sensitivity of LAI around $700-900 \mathrm{~nm}$ (Figure 6) is expected to provide clues for the inversion of LAI for submerged vegetation in shallow-sparse case.

For emergent vegetation, its canopy reflectance is dominated by vegetation parameters, which is similar to the case for terrestrial vegetation. The inversion of LAI will be possible when the vegetation is sparse, while the inversion of the leaf parameters such as Cab is possible in dense cases. The bands around $550 \mathrm{~nm}$ and $710 \mathrm{~nm}$ are peaks for Cab and troughs for LAI, and the band around $670 \mathrm{~nm}$ is a trough for Cab and a peak for LAI, giving suitable conditions for constructing inversion algorithms. These bands have been fully utilized in terrestrial vegetation remote sensing. The above results indicate that it will be a major challenge in wetland remote sensing to distinguish emergent vegetation growing in near shore with terrestrial vegetation [59]. The results for vegetation indices are consistent with those to reflectance in some degree. The sensitivity of LAI to indices is high 
only in shallow cases, whereas the sensitivities of leaf parameters and LIDFa are high only in dense cases. In general, it could be considered that NDVI is better for retrieving LAI in deep-sparse case while NDAVI is suitable in the other three cases. The inversion of leaf parameters in sparse cases is challenging. However, NDVI is useful for inverting Cab and NDAVI is suitable for the inversion of Car in dense cases. The inversion of LIDFa is possible in dense cases by using SAVI or WAVI.

As for submerged vegetation, its reflectance is affected mainly by the water parameters, especially Cchla and Btsm, both of which have significant effects in the entire band range. Compared with the water parameters, the vegetation parameters have a weak influence on the reflectance. Among them, LAI has more influence in the shallow-sparse case while Cab is more sensitive in the shallow-dense case. Almost no vegetation parameters have any significant effect in deep cases. The results to vegetation index are similar. In shallow cases, LAI is the most sensitive vegetation parameter, and the NST to NDAVI is the largest. The sensitivities of LAI and Cab to NDVI in deep cases are relatively higher than to the other indices, which may provide clues for further research. On the other hand, the adjustment factor $L$ in SAVI and WAVI can inhibit the sensitivity to water depth, but failed for water-constituent parameters. This means that the formulae for the adjusted index have yet to be optimized. It may be revealed that for inland or coastal waters containing submerged vegetation, the inversion of vegetation parameters will be more difficult, especially in deep cases. Precise inversion may require considerable priori knowledge. The bands around $550 \mathrm{~nm}, 670 \mathrm{~nm}$ and $710 \mathrm{~nm}$ are characteristic bands for Cab, and the bands around $800 \mathrm{~nm}$ are characteristic bands for LAI. They can provide useful information for algorithm construction.

Either for emergent vegetation or submerged vegetation, the difference between the sensitive patterns of LAI in shallow and dense cases deserves attention. In this study the extent of vegetation cover is described by LAI. For sparse cases, increased LAI means more vegetation signal since LAI is the indicator of the presence and density of vegetation and determines radiation interception by the canopy [36]. So, even a slight change could have a great impact on the canopy reflectance. On the other hand, when the vegetation is dense (LAI > 2), the canopy reflectance tends to be saturated such that it could change only slightly, even if the variation of LAI were obvious. For dense cases, the multiple scatter between leaves is stronger than that of the sparse cases, which means its contribution to canopy reflectance would increase. Leaf parameters such as Cab control mainly the leaf reflectance, which results in their higher sensitivity in dense cases. LIDFa describes the inclination distribution of leaves, which impacts the multiple scatter. Therefore, the variation of LIDFa would easily change the canopy reflectance. In fact, it is similar to the results of Xiao et al. and Villa et al. who observed that when the range of LAI increased, the dominant parameter would change from LAI to Cab [35,38].

It should be emphasized again that the results presented are based on model simulation data; validation of the results may require a large amount of in-situ data, especially data obtained by the variable-controlling approach, which is outside the scope of this paper. Other limitations for this work should be pointed out. First, the global sensitivity analysis simulations are still very expensive computationally. However, parallel processing is a good option to reduce the computation time, since simulations are performed wavelength by wavelength in an independent way. Furthermore, atmospheric effects should be considered to determine the corresponding important parameters for space remote sensing in a future work. To this end, the AVRT model would be integrated with an atmospheric radiative transfer model such as $6 \mathrm{~S}$ or MODTRAN model to deal with the aquatic vegetation-air system. Moreover, the work was limited to the case of statistically independent input parameters. Other possible continuation work could be to consider the case of correlated inputs such as chlorophyll and CDOM concentrations, fluorescence [60].

In fact, it is necessary but difficult, to obtain the accurate measured spectral data for aquatic vegetation, especially submerged vegetation, and corresponding environmental parameters such as LAI, and the concentrations of water constituents. These measured data are significant for research related to inversion and modeling of aquatic vegetation. More standardized and rigorous 
experimental methods and more precise instruments are expected to improve the quality of measured data, and finally to benefit the research in wetland remote sensing.

\section{Conclusions}

The aim of this paper was to identify which target properties are the most significant determinants of spectral reflectance and which ones have a negligible influence in a specific spectral region for aquatic vegetation systems. To this end, a global sensitivity analysis was carried out by the EFAST method with the complex AVRT model to explore a broad range of aquatic vegetation and water body and observation conditions. This work is expected to identify retrievable parameters and provide useful information (priori knowledge) for algorithm constructing in aquatic vegetation remote sensing. The sensitivity to vegetation, water parameters and boundary conditions of canopy reflectance for emergent and submerged vegetation was obtained in four different cases established above. Furthermore, the sensitivities of parameters to the vegetation indices (NDVI, SAVI, NDAVI and WAVI) were obtained to inspire the inversion of aquatic vegetation parameters.

It could be determined whether a parameter might possibly be inverted in some situations by the sensitivity of these parameters in those cases. The results of GSA show that for emergent vegetation, its canopy reflectance is dominated by vegetation parameters, particularly Cab, LAI and LIDFa. The inversion of LAI in sparse cases is possible, while for dense vegetation, the inversion of Cab and LIDFa is also possible. Different from emergent vegetation, the canopy reflectance of submerged vegetation is dominated by water parameters, especially Cchla and Btsm, both of which are highly sensitive over the entire band range. The relatively high sensitivity vegetation parameters are LAI and Cab: they are less influential in deep cases than shallow cases. The sensitivity of Cab has peaks at $550 \mathrm{~nm}$ and $710 \mathrm{~nm}$, while the influence range of LAI is located mainly in 710-820 nm. These wavelength dependencies of sensitivity indices can provide some clues for band selection or band combination, thus aiding retrieval of the most influential parameters. In addition, the sensitivity of LAI in sparse cases is higher than that of Cab, while in dense cases it is the opposite, which is similar to the case for emergent vegetation.

As for the GSA results to VI, in general, NDAVI is suitable for retrieving LAI in cases other than deep-sparse for emergent vegetation, while in that case NDVI would be better. NDVI, NDAVI and WAVI are, respectively, suitable for inverting Cab, Car and LIDFa in dense cases. However, vegetation parameters are less sensitive to these indices for submerged vegetation, especially in deep cases. The sensitivity of LAI to NDAVI is relatively high only in shallow-sparse case, which indicates that NDAVI is difficult to apply in the inversion for submerged vegetation. The analysis results for SAVI and WAVI show that, although the adjustment factor $L$ can resist the sensitivity of water depth, it fails for water constituent parameters such as Cchla and SPM. It should be pointed out that the sensitivity of LAI and Cab to NDVI in deep cases is relatively higher than to the other indices, which may provide justification for further research.

The analysis of this paper points out two future research directions. The first one is to distinguish the emergent vegetation near shore from the terrestrial vegetation by optical characteristics, which would be solved by finding the suitable parameters and their identifiable thresholds. The second one is to construct the submerged vegetation index, which could highlight the vegetation parameters and inhibit the impact of water columns. Currently, it seems difficult to achieve this requirement with spectral information alone. The AVRT model would be expected to play an important role in the future research.

Author Contributions: All authors contributed to the design of the experiment and to its undertaking. All authors revised and approved the final manuscript.

Acknowledgments: This work was supported by the following funds: Beijing Natural Science Foundation (Grant No. 8162028); National Natural Science Foundation of China (Grant No.40901168); CETC key laboratory of aerospace information applications (Grant No. XX17629X009); Beijing Municipal science and technology commission (Grant No. Z171100000717010). We are grateful to Chunyue Niu and Victoria Cheung for their 
constructive help and suggestions. We are also grateful to the anonymous reviewers for their valuable comments and suggestions. This is also a contribution to the activities of the National Centre for Earth Observation of the Natural Environment Research Council (UK).

Conflicts of Interest: The authors declare no conflict of interest.

\section{Appendix}

In the paper of Saltelli et al. [27], the transformation function in Equation (1) was given by:

$$
x_{i}=\frac{1}{2}+\frac{1}{\pi} \arcsin \left(\sin \left(\omega_{i} s+\varphi_{i}\right)\right)
$$

where $\varphi_{i}$ was a random phase-shift determined uniformly in $[0,2 \pi)$. Equation (A1) was proved to take advantage of model simulations efficiently. The variable $s$ was set in the range $(-\pi / 2, \pi / 2)$ and was given by Equation (A2):

$$
s=\frac{\pi}{2}\left(\frac{2 \mathrm{k}-N_{s}-1}{N_{s}}\right), \mathrm{k}=1,2, \cdots N_{s},
$$

where $N_{S}$ was the number of samples required for a single input parameter. It could be determined by $N_{s}=2 M \omega_{\max }+1$, where $M$ was the interference factor which was typically equal to $4, \omega_{\max }$ was the maximum frequency of all the parameters. The setting of $\omega_{i}$ and $\omega_{-i}$ was according to the algorithm in the appendix of Saltelli et al. [61]. After the calculation in Equation (A1) and Equation (A2), each $x_{i}$ was obtained within the range of $0-1$, the actual input samples would be obtained after combining $x_{i}$ with the range and distribution of each input parameter. So the sample size $C$ required by the EFAST method was given by $C=n N_{s}$.

Each input sample was used to generate the model output. After that, the $A_{j}$ and $B_{j}$ would be estimated by:

$$
\begin{gathered}
A_{j}= \begin{cases}\frac{1}{N_{s}}\left\{f\left(\mathrm{~s}_{N_{0}}\right)+\sum_{q=1}^{N_{q}}\left[f\left(\mathrm{~s}_{N_{0}+q}\right)+f\left(\mathrm{~s}_{N_{0}-q}\right)\right] \times \cos \left(\mathrm{j} \frac{\pi}{N_{s}} \mathrm{q}\right)\right\}, & \text { if } j \text { even; } \\
0, & \text { if } j \text { odd. }\end{cases} \\
B_{j}= \begin{cases}0, & \text { if } j \text { even; } \\
\left.\frac{1}{N_{s}}\left\{\sum_{q=1}^{N_{q}}\left[f\left(\mathrm{~s}_{N_{0}+q}\right)-f\left(\mathrm{~s}_{N_{0}-q}\right)\right] \times \sin \left(\mathrm{j} \frac{\pi}{N_{s}} \mathrm{q}\right)\right)\right\}, & \text { if } j \text { odd. }\end{cases}
\end{gathered}
$$

where $N_{q}=\left(N_{s}-1\right) / 2, N_{0}=\left(N_{s}+1\right) / 2$. After that, we could calculate the variances $D_{i}$ and $D$ by Equation (3) and (4). The range of $p$ in Equation (3) was set to $[1, M]$, and the range of $j$ in Equation (4) was $\left[1,\left(N_{S}-1\right) / 2\right]$. The variance $D_{(-\mathrm{i})}$ could be obtained just as in Equation (4) while the range of $j$ was $\left[1, \omega_{i} / 2\right]$. Finally, the first and total order sensitivity index would be obtained by Equation (5) and (6).

\section{References}

1. Ginn, B.K. Distribution and limnological drivers of submerged aquatic plant communities in lake Simcoe (Ontario, Canada): Utility of macrophytes as bioindicators of lake trophic status. J. Great Lakes Res. 2011, 37, 83-89. [CrossRef]

2. Villa, P.; Pinardi, M.; Tóth, V.R.; Hunter, P.D.; Bolpagni, R.; Bresciani, M. Remote sensing of macrophyte morphological traits: Implications for the management of shallow lakes. J. Limnol. 2017, 76. [CrossRef]

3. Cook, C.D.K. Aquatic Plant Book; Quarterly Review of Biology; SPB Academic Publishing: Amsterdam, The Netherlands, 1996. 
4. Hunter, P.D.; Gilvear, D.J.; Tyler, A.N.; Willby, N.J.; Kelly, A. Mapping macrophytic vegetation in shallow lakes using the compact airborne spectrographic imager (casi). Aquat. Conserv. Mar. Freshw. Ecosyst. 2010, 20, 717-727. [CrossRef]

5. Vis, C.; Hudon, C.; Carignan, R. An evaluation of approaches used to determine the distribution and biomass of emergent and submerged aquatic macrophytes over large spatial scales. Aquat. Bot. 2003, 77, 187-201. [CrossRef]

6. Fritz, C.; Dörnhöfer, K.; Schneider, T.; Geist, J.; Oppelt, N. Mapping submerged aquatic vegetation using rapideye satellite data: The example of Lake Kummerow (Germany). Water 2017, 9, 510. [CrossRef]

7. Husson, E.; Reese, H.; Ecke, F. Combining spectral data and a dsm from uas-images for improved classification of non-submerged aquatic vegetation. Remote Sens. 2017, 9, 247. [CrossRef]

8. Luo, J.; Duan, H.; Ma, R.; Jin, X.; Li, F.; Hu, W.; Shi, K.; Huang, W. Mapping species of submerged aquatic vegetation with multi-seasonal satellite images and considering life history information. Int. J. Appl. Earth Obs. Geoinf. 2017, 57, 154-165. [CrossRef]

9. Zhang, X. On the estimation of biomass of submerged vegetation using landsat thematic mapper (tm) imagery: A case study of the Honghu lake, pr China. Int. J. Remote Sens. 1998, 19, 11-20. [CrossRef]

10. Byrd, K.B.; O'Connell, J.L.; Tommaso, S.D.; Kelly, M. Evaluation of sensor types and environmental controls on mapping biomass of coastal marsh emergent vegetation. Remote Sens. Environ. 2014, 149, 166-180. [CrossRef]

11. Gao, Y.; Gao, J.; Wang, J.; Wang, S.; Li, Q.; Zhai, S.; Zhou, Y. Estimating the biomass of unevenly distributed aquatic vegetation in a lake using the normalized water-adjusted vegetation index and scale transformation method. Sci. Total Environ. 2017, 601, 998. [CrossRef] [PubMed]

12. Yadav, S.; Yoneda, M.; Susaki, J.; Tamura, M.; Ishikawa, K.; Yamashiki, Y. A satellite-based assessment of the distribution and biomass of submerged aquatic vegetation in the optically shallow basin of Lake Biwa. Remote Sens. 2017, 9, 966. [CrossRef]

13. Dierssen, H.M.; Zimmerman, R.C.; Leathers, R.A.; Downes, T.V.; Davis, C.O. Ocean color remote sensing of seagrass and bathymetry in the bahamas banks by high-resolution airborne imagery. Limnol. Oceanogr. 2003, 48, 444-455. [CrossRef]

14. Trilla, G.G.; Pratolongo, P.; Beget, M.E.; Kandus, P.; Marcovecchio, J.; Bella, C.D. Relating biophysical parameters of coastal marshes to hyperspectral reflectance data in the Bahia blanca estuary, Argentina. J. Coast. Res. 2013, 29, 231-238. [CrossRef]

15. Everitt, J.H.; Yang, C.; Summy, K.R.; Owens, C.S.; Glomski, L.M.; Smart, R.M. Using in situ hyperspectral reflectance data to distinguish nine aquatic plant species. Geocarto Int. 2011, 26, 459-473. [CrossRef]

16. Pinnel, N.; Heege, T.; Zimmerman, S. Spectral discrimination of submerged macrophytes in lakes using hyperspectral remote sensing data. In Proceedings of the Ocean Optics XVII, Fremantle, Australia, 25-29 October 2004; pp. 1-16.

17. Han, L.; Rundquist, D.C. The spectral responses of ceratophyllum demersum at varying depths in an experimental tank. Int. J. Remote Sens. 2003, 24, 859-864. [CrossRef]

18. Ackleson, S.G.; Klemas, V. Remote sensing of submerged aquatic vegetation in lower chesapeake bay: A comparison of landsat mss to tm imagery. Remote Sens. Environ. 1987, 22, 235-248. [CrossRef]

19. Botha, E.J.; Brando, V.E.; Anstee, J.M.; Dekker, A.G.; Sagar, S. Increased spectral resolution enhances coral detection under varying water conditions. Remote Sens. Environ. 2013, 131, 247-261. [CrossRef]

20. Hedley, J. A three-dimensional radiative transfer model for shallow water environments. Opt. Express 2008, 16, 21887. [CrossRef] [PubMed]

21. Hedley, J.; Roelfsema, C.; Koetz, B.; Phinn, S. Capability of the sentinel 2 mission for tropical coral reef mapping and coral bleaching detection. Remote Sens. Environ. 2012, 120, 145-155. [CrossRef]

22. Sakuno, Y.; Kunii, H. Estimation of growth area of aquatic macrophytes expanding spontaneously in lake Shinji using aster data. Int. J. Geosci. 2013, 4, 1-5. [CrossRef]

23. Suits, G.H. A versatile directional reflectance model for natural water bodies, submerged objects, and moist beach sands. Remote Sens. Environ. 1984, 16, 143-156. [CrossRef]

24. Turpie, K.R. Enhancement of a Canopy Reflectance Model for Understanding the Specular and Spectral Effects of an Aquatic Background in an Inundated Tidal Marsh; University of Maryland: College Park, MD, USA, 2012.

25. Zimmerman, R.C. A biooptical model of irradiance distribution and photosynthesis in seagrass canopies. Limnol. Oceanogr. 2003, 48, 568-585. [CrossRef] 
26. Zhou, G.; Niu, C.; Xu, W.; Yang, W.; Wang, J.; Zhao, H. Canopy modeling of aquatic vegetation: A radiative transfer approach. Remote Sens. Environ. 2015, 163, 186-205. [CrossRef]

27. Saltelli, A.; Tarantola, S.; Chan, P.S. A quantitative model-independent method for global sensitivity analysis of model output. Technometrics 1999, 41,39-56. [CrossRef]

28. Saltelli, A.; Ratto, M.; Andres, T.; Campolongo, F.; Cariboni, J.; Gatelli, D.; Saisana, M.; Tarantola, S. Global Sensitivity Analysis: The Primer; John Wiley \& Sons: Hoboken, NJ, USA, 2008; p. 304.

29. Nossent, J.; Elsen, P.; Bauwens, W. Sobol' Sensitivity Analysis of a Complex Environmental Model; Elsevier Science Publishers B. V.: Amsterdam, Netherlands, 2011; pp. 1515-1525.

30. Saltelli, A.; Marivoet, J. Non-parametric statistics in sensitivity analysis for model output: A comparison of selected techniques. Reliab. Eng. Syst. Saf. 1990, 28, 229-253. [CrossRef]

31. Baroni, G.; Tarantola, S. A general probabilistic framework for uncertainty and global sensitivity analysis of deterministic models: A hydrological case study. Environ. Model. Softw. 2014, 51, 26-34. [CrossRef]

32. Pianosi, F.; Wagener, T. A simple and efficient method for global sensitivity analysis based oncumulative distribution functions. Environ. Model. Softw. 2015, 67, 1-11. [CrossRef]

33. Bounceur, N.; Crucifix, M.; Wilkinson, R.D. Global sensitivity analysis of the climate-vegetation system to astronomical forcing: An emulator-based approach. Earth Syst. Dyn. 2015, 5, 901-943. [CrossRef]

34. Liu, Y.; Chen, K.S. An information entropy-based sensitivity analysis of radar sensing of rough surface. Remote Sens. 2018, 10, 286. [CrossRef]

35. Xiao, Y.; Zhou, D.; Gong, H.; Zhao, W. Sensitivity of canopy reflectance to biochemical and biophysical variables. J. Remote Sens. 2015, 19, 368-374.

36. Mousivand, A.; Menenti, M.; Gorte, B.; Verhoef, W. Global sensitivity analysis of the spectral radiance of a soil-vegetation system. Remote Sens. Environ. 2014, 145, 131-144. [CrossRef]

37. Verrelst, J.; Sabater, N.; Rivera, J.; Muñozmarí, J.; Vicent, J.; Campsvalls, G.; Moreno, J. Emulation of leaf, canopy and atmosphere radiative transfer models for fast global sensitivity analysis. Remote Sens. 2016, 8, 673. [CrossRef]

38. Villa, P.; Mousivand, A.; Bresciani, M. Aquatic vegetation indices assessment through radiative transfer modeling and linear mixture simulation. Int. J. Appl. Earth Obs. Geoinf. 2014, 30, 113-127. [CrossRef]

39. Verrelst, J.; Rivera, J.P.; Tol, C.V.D.; Magnani, F.; Mohammed, G.; Moreno, J. Global sensitivity analysis of the scope model: What drives simulated canopy-leaving sun-induced fluorescence? Remote Sens. Environ. 2015, 166, 8-21. [CrossRef]

40. Jacquemoud, S.; Baret, F. Prospect: A model of leaf optical properties spectra. Remote Sens. Environ. 1990, 34, 75-91. [CrossRef]

41. Bricaud, A.; Babin, M.; Morel, A.E.; Claustre, H.E. Variability in the chlorophyll-specific absorption coefficients of natural phytoplankton: Analysis and parameterization. J. Geophys. Res. 1995, 100, 13321-13332. [CrossRef]

42. Buiteveld, A.H.; Hakvoort, J.H.M.; Donze, M. Optical properties of pure water. In Proceedings of the SPIE-The International Society for Optical Engineering, Bergen, Norway, 13-15 June 1994; pp. 368-373.

43. Hr, P.A.S.; Markager, S. Parameterization of the chlorophyll a-specific in vivo light absorption coefficient covering estuarine, coastal and oceanic waters. Int. J. Remote Sens. 2004, 25, 5117-5130.

44. Palmer, K.F.; Williams, D. Optical properties of water in the near infrared. J. Opt. Soc. Am. 1974, 64, 1107-1110. [CrossRef]

45. Smith, R.C.; Baker, K.S. Optical properties of the clearest natural waters (200-800 nm). Appl. Opt. 1981, 20, 177-184. [CrossRef] [PubMed]

46. Volpe, V.; Silvestri, S.; Marani, M. Remote sensing retrieval of suspended sediment concentration in shallow waters. Remote Sens. Environ. 2011, 115, 44-54. [CrossRef]

47. Cox, C.; Munk, W. Measurement of the roughness of the sea surface from photographs of the sun's glitter. J. Opt. Soc. Am. 1954, 44, 838-850. [CrossRef]

48. Cooper, K.; Smith, J.A.; Pitts, D. Reflectance of a vegetation canopy using the adding method. Appl. Opt. 1982, 21, 4112-4118. [CrossRef] [PubMed]

49. Verhoef, W. Light scattering by leaf layers with application to canopy reflectance modeling: The sail model. Remote Sens. Environ. 1984, 16, 125-141. [CrossRef] 
50. Babin, M.; Stramski, D.; Ferrari, G.M.; Claustre, H.; Bricaud, A.; Obolensky, G.; Hoepffner, N. Variations in the light absorption coefficients of phytoplankton, nonalgal particles, and dissolved organic matter in coastal waters around europe. J. Geophys. Res. Oceans 2003, 108. [CrossRef]

51. Lee, Z.; Carder, K.L.; Mobley, C.D.; Steward, R.G.; Patch, J.S. Hyperspectral remote sensing for shallow waters. I. A semianalytical model. Appl. Opt. 1998, 37, 6329. [CrossRef] [PubMed]

52. Dimitris, S.; Heiko, B.; Olga, S.; András, Z.; Tóth, V.R. Evaluating sentinel-2 for lakeshore habitat mapping based on airborne hyperspectral data. Sensors 2015, 15, 22956-22969.

53. Dall'Olmo, G.; Gitelson, A.A. Effect of bio-optical parameter variability on the remote estimation of chlorophyll-a concentration in turbid productive waters: Experimental results. Appl. Opt. 2005, 44, 412-422. [CrossRef] [PubMed]

54. Le, C.; Li, Y.; Zha, Y.; Sun, D.; Huang, C.; Lu, H. A four-band semi-analytical model for estimating chlorophyll a in highly turbid lakes: The case of Taihu Lake, china. Remote Sens. Environ. 2009, 113, 1175-1182. [CrossRef]

55. Huete, A.; Justice, C.; Liu, H. Development of vegetation and soil indices for modis-eos. Remote Sens. Environ. 1994, 49, 224-234. [CrossRef]

56. Bannari, A.; Morin, D.; Bonn, F.; Huete, A.R. A review of vegetation indices. Remote Sens. Rev. 1995, 13, 95-120. [CrossRef]

57. Huete, A.R. A soil-adjusted vegetation index (savi). Remote Sens. Environ. 1988, 25, 295-309. [CrossRef]

58. Huete, A.R.; Liu, H.Q.; Batchily, K.; Leeuwen, W.V. A comparison of vegetation indices over a global set of tm images for eos-modis. Remote Sens. Environ. 1997, 59, 440-451. [CrossRef]

59. Silva, T.S.; Costa, M.P.; Melack, J.M.; Novo, E.M. Remote sensing of aquatic vegetation: Theory and applications. Environ. Monit. Assess. 2008, 140, 131-145. [CrossRef] [PubMed]

60. Duarte-Carvajalino, J.M. Sensitivity Analysis of the Water Column Effect on the Remote Sensing Reflectance, the Shallow Waters Case. Master's Thesis, University of Puerto Rico, Mayaguez, Puerto Rico, 2003.

61. Saltelli, A.; Annoni, P. Sensitivity Analysis; Wiley: Hoboken, NJ, USA, 2000; pp. 1298-1301.

(c) 2018 by the authors. Licensee MDPI, Basel, Switzerland. This article is an open access article distributed under the terms and conditions of the Creative Commons Attribution (CC BY) license (http:// creativecommons.org/licenses/by/4.0/). 